\title{
Reubicándose en la universidad. Propuesta de una tasa de reubicaciones a partir de un estudio de trayectorias educativas
}

\author{
Relocating within the university. Proposal of a relocation rate based on a study of educational \\ trajectories
}

\author{
Villar-Aguilés, Alícia; Hernàndez i Dobon, Francesc Jesús \& García-Ros, Rafael \\ Universidad de Valencia (España)
}

\begin{abstract}
Resumen
La universidad ha vivido transformaciones importantes en los últimos años como es la expansión de una mayor heterogeneidad en el perfil de los estudiantes. Esta realidad ha implicado cambios significativos en el acceso a la universidad, los resultados y la salida hacia el mercado laboral de los universitarios. Los estudiantes provienen de entornos socioeconómicos diferentes, de vías de acceso diversas y se incorporan a la universidad con distintas expectativas y ritmos están produciendo una diversificación de las trayectorias educativas. En este sentido, esta diversificación conduce a una mayor frecuencia de trayectorias que desencadenan cambios de grado y abandonos de los estudios, lo que ha supuesto que se hayan desarrollado numerosos trabajos para determinar los factores explicativos. Este trabajo aborda los cambios de grados y abandonos de los estudios universitarios utilizando el concepto de "reubicación académica” mediante un análisis de trayectorias de 60.300 sujetos. El período analizado ha sido seis de cursos académicos (2009-10 a 2014-15). El objetivo es estudiar las "reubicaciones" entre titulaciones de grado para, a partir de ello, proponer el cálculo de un nuevo indicador: la "tasa de reubicaciones". Las diferencias apreciadas entre titulaciones y su agrupación por ramas de conocimiento permiten constatar la importante heterogeneidad institucional.
\end{abstract}

Palabras clave:

Estudios universitarios, estudiantes, rendimiento, abandono, trayectorias educativas

\begin{abstract}
The university has undergone important changes in recent years as the expansion of greater heterogeneity in the student profile. This reality has led to significant changes in the university access, the achievement and the exit to the labour market. Students from different socioeconomic backgrounds, different access pathways and incorporated into the university with different expectations and rhythms are producing a diversification of educational paths. In this sense, a higher frequency of educational pathways that trigger changes and school leavers occurs, which has meant that numerous studies have been developed to determine the explanatory factors. This article approaches the changes of degrees and dropouts of university studies using the concept of "academic relocation" through an analysis of educational pathways of 60,300 subjects. The period examined have been six academic years (2009-10 to 201415). The main aim is to study the "relocations" between undergraduate degrees for, from this, proposes a new indicator: the "rate of relocations". The perceived differences between degrees and their grouping by branches of knowledge allow noting the important institutional heterogeneity.
\end{abstract}

\section{Keywords:}

University studies, students, achievement, dropout, educational pathways

Reception Date

2016 October 10

Approval Date

2017 June 15

Publication Date: 2017 June 15 
La idea de una universidad homogénea, con un estudiantado proveniente de un segmento o clase social similar y con un comportamiento académico mayoritariamente análogo, en cuanto a la dedicación a los estudios, forma parte del pasado. En las décadas previas a la denominada democratización de la universidad también existían estudiantes con distintos orígenes sociales y con ritmos de estudios y dedicaciones diferentes, pero en las últimas dos décadas la universidad española ha vivido importantes transformaciones, con un estudiantado más heterogéneo y que presenta índices altos de abandono y prolongación de estudios.

Algunas investigaciones previas dedicadas al fenómeno del abandono de estudios universitarios muestran que existe una importante variación por titulaciones (Cabrera, Bethencourt, Álvarez Pérez \& González Afonso, 2006) y que se trata de un fenómeno multidimensional que debe ser estudiado desde diferentes ópticas y disciplinas de manera complementaria. Los estudios más tradicionales y comunes en este campo se han centrado en analizar el nivel de asociación y la capacidad predictiva sobre el éxito académico de las variables sociodemográficas, educativas previas al acceso y relativas a circunstancias personales de los estudiantes, como el género, la dedicación a los estudios, los resultados académicos previos, el estatus sociofamiliar u origen social, el grupo sociocultural de pertenencia o las dificultades económicas, entre otras, $\mathrm{y}$, habitualmente, a través de la comparación entre sujetos que abandonan y sujetos que continúan sus estudios hasta obtener su titulación (Glogowska, Young \& Lockyer, 2007). Entre sus conclusiones fundamentales destaca que los resultados en etapas educativas previas $\mathrm{y}$ en pruebas estandarizadas de rendimiento efectuadas antes de ingresar en la universidad, así como el estatus socioeconómico familiar constituyen predictores significativos del éxito en la universidad (Bethencourt, Cabrera, Hernández, Álvarez \& González, 2008; Lassibille \& Navarro, 2009; Rodrigo, Molina, García-Ros \& Pérez González, 2012), resultando especialmente importante identificar y ofrecer respuestas diferenciales a las problemáticas y necesidades de los estudiantes que acceden a las aulas universitarias con distintas composiciones sociales y que generan efectos diversos (Ariño \& Llopis, 2011; Fachelli \& Navarro, 2015; Troiano \& Elías, 2013; Villar, 2010).

Recientemente, el Anuario de Indicadores Universitarios que publica el Ministerio de Educación (2016) ha incorporado un nuevo indicador que contabiliza el cambio de estudios: la "tasa de cambio de estudio", que muestra el porcentaje de estudiantes de una cohorte de nuevo ingreso en un curso determinado matriculado en una titulación y en una universidad y que no se han matriculado en esa misma titulación durante dos cursos seguidos, porque se han matriculado en algún otro título y no se han graduado. Esta novedad matiza la cuantificación del abandono, ya que hasta entonces "abandono" y "cambio" no se distinguían y todo cambio de titulación se computaba como abandono, lo cual no era del todo preciso o, al menos, era cuestionable.

El abandono global (suma de las tasas parciales de abandono del estudio en primer, segundo y tercer año) para el total de universidades públicas se sitúa en un $37,5 \%$, siendo significativa la diferenciación en mujeres y hombres, puesto que ellos abandonan más (40,5\%) que ellas (31,6\%). En las universidades públicas valencianas este dato se encuentra en un $27,3 \%$, siendo también significativa la diferencia entre hombres (31,3\%) y mujeres (23,7\%).

En cuanto a la tasa parcial de abandono, los datos disponibles referidos a la cohorte de entrada 2010-2011 presentan unos porcentajes de abandono en primer, segundo y tercer año del $19,6 \%$, el $7,9 \%$ y el $4,0 \%$ respectivamente referidos a mujeres, y del 24,9\%, 10,5\% y $5,1 \%$ referidos a hombres, por lo que respecta a las universidades públicas, a efectos de comparar con el estudio realizado.

La tasa de cambio de estudio presenta porcentajes menores que la de abandono, situándose en un 13\% para el caso de las 
universidades públicas españolas; esta tasa también es más acentuada en el caso de los hombres (15,39\%), que en las mujeres (10,92\%). En el caso de las universidades públicas valencianas la cifra desciende a $11,69 \%$.

En trabajos anteriores ya habíamos apuntado la conveniencia de considerar de manera diferenciada cambio de titulación respecto a abandono de estudios universitarios (Villar, Vieira, Hernàndez \& Almeida, 2012). Por tanto, consideramos que este reciente indicador considerado por el Ministerio amplia y mejora la información sobre trayectorias formativas del estudiantado universitario. Aun así, en el presente trabajo planteamos un nuevo indicador que aspira a complementar los dos anteriormente mencionados, con un cálculo que focaliza hacia las titulaciones, más que al estudiantado; lo denominamos la "tasa de reubicaciones".

La tasa de cambio del estudio considera como unidad de medida el número de estudiantes que cambia de carrera. La tasa que proponemos, la "tasa de reubicaciones", considera como unidad de medida la titulación, es decir, enfoca la mirada no hacia los estudiantes, sino hacia las titulaciones. Así pues, el objetivo es cuantificar los movimientos de matrícula que tienen las titulaciones, tanto en un sentido de atracción como de expulsión. Partimos de la idea, apoyada por nuestras investigaciones, de que existen titulaciones universitarias que, estructuralmente, contienen una mayor probabilidad de reubicaciones que otras. Los factores que inciden en ello son diversos. Por tanto, esta tasa no contradice la tasa de cambio del estudio propuesta por el Ministerio, sino que la complementa.

En la universidad actual podemos afirmar que los itinerarios formativos no transcurren necesariamente de manera lineal, ni unívoca. Existen itinerarios formativos de retorno, de reubicación. Las trayectorias educativas se cuestionan y replantean $\mathrm{y}$ las decisiones educativas se reubican. El concepto de "reubicación" nos permite ampliar la mirada para entender las transiciones formativas desde una posición analítica que considera la reversibilidad y la no linealidad como definitorias de una gran parte de las actuales trayectorias formativas, y también laborales de los jóvenes.

De manera sintetizada, el objetivo de este texto se concreta en estudiar las reubicaciones entre titulaciones, para llegar al cálculo de una "tasa de reubicaciones". Las diferencias apreciadas entre titulaciones y su agrupación por ramas de conocimiento permiten constatar la importante heterogeneidad institucional.

\section{Método}

\section{Participantes}

El contexto en el que se ha realizado esta investigación es la Universidad de Valencia. Los datos proceden de una explotación diseñada y realizada expresamente de seis bases de datos correspondientes a la matrícula en primer curso desde el curso 2009-10 hasta 2014-15. Hemos obtenido datos de estudiantes que se han matriculado en una, dos, tres y hasta en cuatro ocasiones en primer curso durante este período de seis cursos. En total, el número de sujetos considerados han sido 60.300, que han generado 63.488 matrículas.

\section{Procedimiento}

En primer lugar, realizamos un análisis descriptivo de datos sobre reubicaciones desagregando según el número de matrículas registradas y ramas de conocimiento. Seguidamente, presentamos una matriz de reubicaciones para observar gráficamente las transiciones entre titulaciones. En tercer lugar, calculamos la pauta general que siguen las reubicaciones estudiadas mediante una ecuación. A continuación, se muestran los datos sobre la tasa de reubicaciones, una propuesta nueva que focaliza el cálculo teniendo en cuenta la proporción de grados de una universidad.

Es pertinente advertir que las bases de datos utilizadas no permiten discriminar aquellas matrículas que son segundas o 
sucesivas matrículas de estudiantado que ya se había matriculado con anterioridad al primer año académico considerado, es decir, antes de 2009-10. Aún así, el hecho de incluir datos de seis cursos con un conjunto numeroso de estudiantado nos permite reducir el posible efecto estadístico del estudiantado matriculado anteriormente a 2009.

\section{Resultados}

En la Universidad de Valencia se publican indicadores de los grados y los másteres impartidos sobre el desarrollo de la enseñanza, como es la tasa de abandono de los estudiantes de primer curso. Esta tasa, de cálculo estandarizado a nivel estatal y compartido por el resto de universidades, muestra, como es sabido, el porcentaje de estudiantes que habiéndose matriculado un año en la universidad no lo han hecho en los dos cursos posteriores. También se calcula el indicador denominado cambio de estudios, como ya hemos comentado en el apartado de introducción a este texto. Estos indicadores focalizan el cálculo en los estudiantes, es decir, toman como unidad de medida a los estudiantes que abandonan o cambian de carrera sin ninguna otra consideración. Por ello, nos planteamos considerar el fenómeno de cambios de trayectorias académicas de los estudiantes a partir del concepto de reubicación, se trata de otra semántica que matiza los indicadores convencionales. Las reubicaciones según las entendemos aquí no focalizan en el estudiante, sino que giran la mirada hacia las titulaciones, hacia la rama de conocimiento, el momento del cambio y otras apreciaciones. No es tanto el estudiante quien se reubica, en su individualidad, sino que existiría un mapa de reubicaciones que sigue una pauta general en la institución estudiada.

Reubicaciones: datos descriptivos básicos

Primeramente, observamos los datos sobre reubicaciones desagregados según el número de matrículas realizadas (tabla 1 ).

Tabla 1. Personas y matrículas registradas. Cursos 2009-10 al 2014-15

\begin{tabular}{|c|c|c|c|c|c|c|}
\hline \multicolumn{3}{|c|}{ Estudiantado matriculado en primer curso } & $\begin{array}{c}\text { Personas } \\
\text { matriculadas }\end{array}$ & Porcentaje & $\begin{array}{c}\text { Matrículas } \\
\text { realizadas }\end{array}$ & Porcentaje \\
\hline \multicolumn{3}{|l|}{$1 \mathrm{vez}$} & 57225 & $94,90 \%$ & 57225 & $90,14 \%$ \\
\hline \multirow{2}{*}{2 veces } & En el mismo curso & 476 & \multirow{2}{*}{2967} & \multirow{2}{*}{$4,92 \%$} & \multirow{2}{*}{5934} & \multirow{2}{*}{$9,35 \%$} \\
\hline & En cursos distintos & 2491 & & & & \\
\hline \multicolumn{3}{|l|}{3 veces } & 103 & $0,17 \%$ & 309 & $0,49 \%$ \\
\hline \multicolumn{3}{|l|}{4 veces } & 5 & $0,01 \%$ & 20 & $0,03 \%$ \\
\hline \multicolumn{3}{|c|}{ Reubicaciones } & 3075 & $5,10 \%$ & 6263 & $9,86 \%$ \\
\hline \multicolumn{3}{|l|}{ Total } & 60300 & $100 \%$ & 63488 & $100 \%$ \\
\hline
\end{tabular}

Fuente: Elaboración propia bases de datos matrícula primer curso 2009-10 al 2014-15.

Las reubicaciones afectan aproximadamente a un $5 \%$ del estudiantado o a un $10 \%$ de las matrículas. A continuación, estudiaremos con más detalle este fenómeno.

Para poder estudiar con más detalle la dinámica de las reubicaciones, hemos tenido en cuenta algunas decisiones metodológicas. En primer lugar, prescindimos en los análisis siguientes de las personas que acumulan tres y cuatro matrículas en primer curso, que son 103 y 5 respectivamente (ver tabla 1 ), porque en estos casos existe una casuística heterogénea de titulaciones de origen y de destino. Es por ello que estudiaremos únicamente al grupo de personas que en los cursos considerados se han vuelto a matricular una vez en primer curso, es decir, un total de 2.967 personas que han generado 5.934 matrículas.

En segundo lugar, de este total de personas prescindiremos de 476 que se han vuelto a matricular en primer curso en el mismo curso académico; esto se debe a dos motivos, porque 
Villar Aguilés, Alicia; Hernàndez i Dobon, Francesc Jesús \& García Ros, Rafael (2017). Reubicándose en la universidad. Propuesta de una tasa de reubicaciones a partir de un estudio de trayectorias educativas. RELIEVE, 23(1), art. 5. doi: http://doi.org/10.7203/relieve.23.1.9059

se trate de reajustes administrativos, por ejemplo, por reasignaciones de plazas posteriores a un primer período de matrícula (aquello que conocemos popularmente como "subasta de plazas"), y porque las bases de datos analizadas no permiten distinguir cuándo fue cronológicamente la primera matrícula y cuál la segunda. La muestra, por tanto, se reduce a 2.491 personas.

En tercer lugar, también prescindiremos de 32 personas que se volvieron a matricular cinco años después de la primera matrícula, lo que puede tratarse de reubicación o matrícula en un grado después de concluir otro. De esta manera, la muestra estudiada, definitivamente, está conformada por 2.459 personas.

A continuación, se muestran los datos del análisis del flujo de reubicaciones según rama de conocimiento, a la cual están adscritos los grados universitarios. En la tabla 2 se observa el detalle de la rama de la primera matrícula (filas) y la rama de la segunda matrícula (columnas). Seguidamente, en la tabla 3, se reelaboran estos datos para distinguir las reubicaciones realizadas en titulaciones fuera de la misma rama, que denominaremos ad extra, y dentro de la misma rama, que denominaremos ad intra.

Tabla 2. Reubicaciones según ramas (valores absolutos)

\begin{tabular}{lccccccccc}
\hline & AI & AH & C & $\begin{array}{c}\text { Centro } \\
\text { adscrito }\end{array}$ & S & SJ & Total & Saldo \\
\hline AI & 62 & 5 & 6 & 0 & 6 & 24 & 103 & -6 & $-5,82 \%$ \\
\hline AH & 2 & 233 & 5 & 3 & 7 & 130 & 380 & 17 & $4,47 \%$ \\
\hline C & 11 & 17 & 61 & 2 & 84 & 64 & 239 & -116 & $-48,53 \%$ \\
\hline Centro adscrito & 1 & 4 & 0 & 265 & 3 & 109 & 382 & -80 & $-20,94 \%$ \\
\hline S & 0 & 14 & 37 & 2 & 215 & 75 & 343 & 10 & 2,91 \\
\hline SJ & 21 & 124 & 14 & 30 & 38 & 785 & 1012 & 175 & $17,29 \%$ \\
\hline Total & 97 & 397 & 123 & 302 & 353 & 1187 & 2459 & & \\
\hline
\end{tabular}

Fuente: Elaboración propia bases de datos matrícula primer curso 2009-10 al 2014-15.

AI: Arquitectura-Ingeniería; AH: Artes y Humanidades; C: Ciencias; S: Ciencias Salud; SJ: Ciencias Sociales y Jurídicas

Tabla 3. Reubicaciones ad extra y ad intra de las ramas (valores absolutos y relativos)

\begin{tabular}{lccccccc}
\hline & $\begin{array}{c}\text { Total matrículas } \\
\text { realizadas }\end{array}$ & Total & Proporción & $\begin{array}{c}\text { Ad } \\
\text { extra }\end{array}$ & $\begin{array}{c}\text { \% Ad } \\
\text { extra }\end{array}$ & $\begin{array}{c}\text { Ad } \\
\text { intra }\end{array}$ & $\begin{array}{c}\text { \% Ad } \\
\text { intra }\end{array}$ \\
\hline AI & 2368 & 103 & $4,35 \%$ & 41 & $39,81 \%$ & 62 & $60,19 \%$ \\
\hline AH & 8834 & 380 & $4,30 \%$ & 147 & $38,68 \%$ & 233 & $61,32 \%$ \\
\hline C & 5422 & 239 & $4,41 \%$ & 178 & $74,48 \%$ & 61 & $25,52 \%$ \\
\hline $\begin{array}{l}\text { Centro } \\
\text { adscrito }\end{array}$ & 2279 & 382 & $16,76 \%$ & 117 & $30,63 \%$ & 265 & $69,37 \%$ \\
\hline S & 11963 & 343 & $2,87 \%$ & 128 & $37,32 \%$ & 215 & $62,68 \%$ \\
\hline SJ & 32614 & 1012 & $3,10 \%$ & 227 & $22,43 \%$ & 785 & $77,57 \%$ \\
\hline No consta & 8 & & & & & & \\
\hline Total & 63488 & 2459 & $3,87 \%$ & 838 & $34,08 \%$ & 1621 & $65,92 \%$ \\
\hline
\end{tabular}

Fuente: Elaboración propia a partir de las bases de datos sobre matrícula primer curso 2009-10 a 2014-15.

AI: Arquitectura-Ingeniería; AH: Artes -Humanidades; C: Ciencias; S: Ciencias Salud; SJ: Ciencias Sociales y Jurídicas

En general, dos tercios de las reubicaciones son ad intra, es decir, se producen dentro de la misma rama de conocimiento, y un tercio son ad extra. El valor más elevado lo presenta
Ciencias Sociales y Jurídicas, con un 77,57\% ad intra; el valor más bajo se observa en Ciencias, con un 74,48\% ad extra. Dicho de otra forma, en Ciencias Sociales y Jurídicas 
solo 1 de cada 4 reubicaciones son a otra rama, mientras que en Ciencias el comportamiento es el inverso: solamente 1 de cada 4 reubicaciones se mantiene en la misma rama.

Este comportamiento diferenciado se refleja también en el cálculo del saldo de las reubicaciones. La diferencia neta respecto del volumen de reubicaciones proporciona porcentajes elevados y positivos en el caso de Ciencias Sociales y Jurídicas (17,29\%) y muy elevados y negativos en Ciencias (48,53\%). En cuanto, al número de reubicaciones respecto al conjunto de matrículas (consideradas en conjunto, es decir, sin descontar las matrículas que son segundas, terceras o cuartas matrículas de estudiantado reubicado), se aprecia una proporción importante de reubicaciones en casos de centros adscritos a la Universidad de Valencia. Dicho caso exigiría un estudio particularizado. El número menor de reubicaciones lo encontramos proporcionalmente en Ciencias de la Salud, seguido de Ciencias Sociales y Jurídicas. Arquitectura e Ingeniería, Artes y Humanidades y Ciencias presentan porcentajes similares, aunque, como se ha señalado, hay una diferencia notable entre la proporción de reubicaciones ad intra y ad extra en el caso de Ciencias.

Analizaremos ahora el momento de la reubicación. Los análisis se centran en determinar el número de casos en que se ha producido un cambio de titulación donde se había realizado la matrícula (grados de origen) en el período indicado (2009/10 hasta 2014/15) y donde se han vuelto a matricular una vez en otra titulación (grados de destino); en función del curso en el que hicieron el cambio desde la primera matrícula. Se han descartado los casos codificados con 0 , porque pueden deberse a casos de reajustes administrativos de matrícula en el mismo año, y los casos superiores a 4 porque pueden deberse a casos en que se ha finalizado una titulación y se inicia una segunda.

\section{Matriz de reubicaciones}

Los resultados destacan que existen importantes diferencias entre salidas $\mathrm{y}$ entradas en cada grado. Los datos sobre la diferencia entre salidas y entradas, en términos absolutos, y la relación porcentual del incremento muestran aumentos muy altos y negativos lo que significa que en esas titulaciones se ha dado una salida importante de estudiantes, ya que en algunos casos se llega a superar el $50 \%$.

De manera sintetizada, en la tabla 4 se pueden observar las reubicaciones por rama de conocimiento y según el año del cambio desde la primera matrícula en la siguiente tabla.

Tabla 4. Reubicaciones según ramas de conocimiento, en valores absolutos y relativos (porcentajes horizontales)

\begin{tabular}{lccccccccc}
\hline & \multicolumn{4}{c}{ Año del cambio } & \multicolumn{4}{c}{ Año del cambio } \\
\hline Rama & $\mathbf{1}$ & $\mathbf{2}$ & $\mathbf{3}$ & $\mathbf{4}$ & Total & $\mathbf{1}$ & $\mathbf{2}$ & $\mathbf{3}$ & $\mathbf{4}$ \\
Arquitectura e Ingeniería & 66 & 20 & 13 & 4 & 103 & $64,08 \%$ & $19,42 \%$ & $12,62 \%$ & $3,88 \%$ \\
Artes y Humanidades & 281 & 55 & 19 & 25 & 380 & $73,95 \%$ & $14,47 \%$ & $5,00 \%$ & $6,58 \%$ \\
Ciencias & 160 & 43 & 26 & 10 & 239 & $66,95 \%$ & $17,99 \%$ & $10,88 \%$ & $4,18 \%$ \\
Centros adscritos & 339 & 31 & 10 & 2 & 382 & $88,74 \%$ & $8,12 \%$ & $2,62 \%$ & $0,52 \%$ \\
Ciencias de la Salud & 217 & 75 & 26 & 25 & 343 & $63,27 \%$ & $21,87 \%$ & $7,58 \%$ & $7,29 \%$ \\
Ciencias Sociales y Jurídicas & 726 & 147 & 83 & 56 & 1012 & $71,74 \%$ & $14,53 \%$ & $8,20 \%$ & $5,53 \%$ \\
Total & 1789 & 371 & 177 & 122 & 2459 & $72,75 \%$ & $15,09 \%$ & $7,20 \%$ & $4,96 \%$ \\
\hline
\end{tabular}

Fuente: Elaboración propia bases de datos matrícula primer curso 2009-10 al 2014-15. 


\section{Ecuación de reubicaciones}

Estos datos nos llevan a calcular la pauta general de las reubicaciones de los grados para el caso de estudio de la Universidad de Valencia, expresada mediante la línea de tendencia. Dicha pauta sigue una línea exponencial y se puede representar con la siguiente ecuación:

$$
\text { [1] } \quad y_{n}=\frac{2}{3} x_{n}^{-2}
$$

Esta ecuación de reubicaciones indica la proporción de estudiantado que se reubica $\left(y_{n}\right)$ en función del curso $\left(x_{n}\right), y$ es una aproximación razonable a la fórmula $\mathrm{y}_{\mathrm{n}}=$ $0,6717 \mathrm{x}_{\mathrm{n}}{ }^{-1,967}$, que corresponde a la ecuación de la línea de tendencia de los valores de la tabla 5, y que será tomada aquí como base provisional. Así, para el caso estudiado, los porcentajes de reubicación a partir del curso serían:

Tabla 5. Porcentajes de reubicación por curso

\begin{tabular}{ccc}
\hline Curso & $\mathrm{y}$ & acumulado \\
\hline 1 & $66,66 \%$ & $66,66 \%$ \\
2 & $16,66 \%$ & $83,33 \%$ \\
3 & $7,40 \%$ & $90,74 \%$ \\
4 & $4,16 \%$ & $94,90 \%$ \\
\hline
\end{tabular}

Esto significa que, al disponer de una ecuación de reubicaciones, no es preciso establecer el cómputo total de las reubicaciones, puesto que se puede efectuar una estimación a partir de un único curso. Lógicamente, el total de reubicaciones corresponde a la fórmula :

$$
\text { [2] } \quad r_{t}=\frac{r_{x}}{y_{n}}
$$

A efectos administrativos, resulta trivial que

$$
r_{x}=\frac{m_{2}}{2}+\frac{m_{\mathrm{a}}}{3}+\cdots
$$

Es decir, el número de reubicaciones es igual a la suma del número de matrículas de las personas que se han matriculado dos veces en un curso dividida por dos, las matrículas realizadas por las personas que se han matriculado tres veces dividida por tres, etc. Esta afirmación es precisa para evitar el efecto de duplicaciones en el cómputo final.

Dejando a parte el caso de los grados que se imparten en centros adscritos a la Universidad de Valencia, porque presentan un comportamiento extremo, las líneas de tendencia de las ramas de conocimiento, en las que se agrupan los grados, presentan diferencias entre si. Cabe decir que las líneas de tendencia exponencial representan mejor que cualquier otra la situación de los valores. Por su parte, las líneas de tendencia recta simplifican esta representación mostrando la pendiente de las líneas, tal y como se recoge en la tabla 6.

Tabla 6. Líneas de tendencia por ramas de conocimiento

\begin{tabular}{l|c|c|}
\hline & Línea de tendencia exponencial & Línea de tendencia recta \\
\hline Total & $\mathrm{y}=0,6717 \mathrm{x}-1,967$ & $\mathrm{y}=-0,2113 \mathrm{x}+0,7782$ \\
Arquitectura e Ingeniería & $\mathrm{y}=0,6966 \mathrm{x}-1,874$ & $\mathrm{y}=-0,1874 \mathrm{x}+0,7184$ \\
Artes y Humanidades & $\mathrm{y}=0,6316 \mathrm{x}-1,924$ & $\mathrm{y}=-0,2116 \mathrm{x}+0,7789$ \\
Ciencias & $\mathrm{y}=0,6925 \mathrm{x}-1,9$ & $\mathrm{y}=-0,1954 \mathrm{x}+0,7385$ \\
Ciencias de la Salud & $\mathrm{y}=0,6293 \mathrm{x}-1,675$ & $\mathrm{y}=-0,1822 \mathrm{x}+0,7055$ \\
Ciencias Sociales y Jurídicas & $\mathrm{y}=0,6451 \mathrm{x}-1,857$ & $\mathrm{y}=-0,2049 \mathrm{x}+0,7624$ \\
\hline
\end{tabular}

Si nos fijamos en el factor $a$ de la ecuación de la línea de tendencia nos permite ordenar las ramas de conocimiento según la siguiente disposición: 


$$
\text { AH - Total - SJ - C - AI - S }
$$

En un extremo tenemos la rama de Arte y Humanidades (AH) que presenta una pendiente (descendiente) mayor, lo que significa que la frecuencia de reubicaciones será relativamente mayor a la media durante los primeros años de matrícula universitaria. En el otro extremo aparece Ciencias de la Salud (S), una rama en la que las reubicaciones tendrán lugar pasados los primeros años universitarios.

Gráfico 1. Pauta de comportamiento de las reubicaciones por ramas

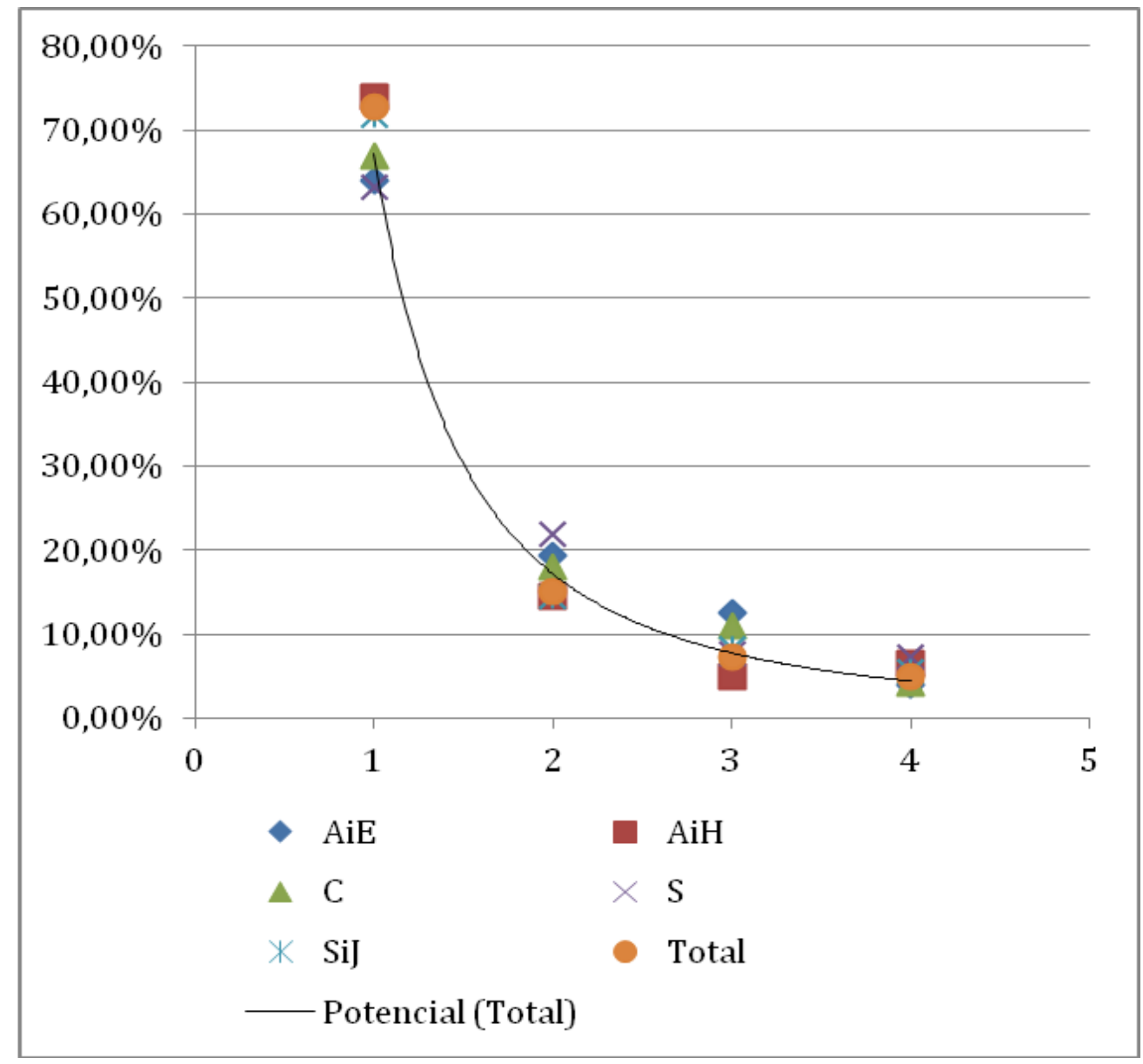

\section{Tasa de reubicaciones}

A partir de las fórmulas que nos permiten llegar a la ecuación de reubicaciones se puede calcular una tasa de reubicaciones $(T R)$, para una universidad concreta $(u)$ que oferte una serie de grados $(g)$ y cuente con un estudiantado en un curso $\left(e_{x}\right)$, para un curso académico concreto, que podríamos definir de este modo:

[4]

$$
T R_{u}=\frac{r_{x}}{y_{n} e_{x} \ln (g-1)}
$$

El factor $\ln (g-1)$ en el denominador pretende matizar el hecho de que las reubicaciones son posibles en mayor proporción en aquellas universidades con una oferta de títulos mayor. Lógicamente podría utilizarse cualquier otro factor corrector.

$\mathrm{Y}$ en forma extendida (utilizando la ecuación general de la fórmula [1])

$$
T R_{u}=\frac{3 x^{2}\left(\frac{m_{2}}{2}+\frac{m_{3}}{3} \ldots\right)}{2 e_{x} \ln (g-1)}
$$


Que para el caso del primer curso, queda:

$$
T R_{u}=\frac{3\left(\frac{m_{2}}{2}+\frac{m_{3}}{3} \ldots\right)}{2 e_{x} \ln (g-1)}
$$

Lógicamente, para un uso administrativo sería preciso recalcular la fórmula [1] para un conjunto de universidades mayor.

\section{Discusión}

En este trabajo avanzamos en el estudio del abandono o cambio de los estudios universitarios, pero con una nueva propuesta semántica y de cálculo como es el concepto de reubicaciones acompañado de expresiones matemáticas, tal y como hemos mostrado mediante la propuesta de una tasa de reubicaciones.

El estudio de reubicaciones, entendidas de manera más amplia a la cuantificación del abandono universitario o del cambio de estudios, muestra las diferencias significativas que existen dentro de una misma institución universitaria puesto que los resultados son diferentes según titulaciones de grado y según su agrupación por ramas de conocimiento.

Los datos que hemos analizado incorporan información de seis cursos distinguiéndose entre las reubicaciones por ramas de conocimiento, por titulaciones y según el momento en que se producen los movimientos.

Las transiciones entre grados y de manera ad intra y ad extra, es decir, dentro y fuera de la rama de conocimiento, permiten constatar la fuerte heterogeneidad existente en el estudio de caso para la Universidad de Valencia. En general, dos tercios de las reubicaciones son ad intra y un tercio son ad extra.

La observación de los cálculos nos llevan a pensar que las posibles o futuras estrategias de intervención que podrían plantearse en la institución universitaria deberían ser diferenciadas según titulaciones 0 por agrupaciones de algunos grados que presentan comportamientos similares o, al menos, según las agrupaciones por ramas de conocimiento.
En este sentido, las actuaciones que podrían implementarse en Artes y Humanidades, por mencionar un caso, serían parcialmente diferentes de las que se aplicarían en Ciencias de la Salud. En el caso de las ramas de Artes y Humanidades y de Ciencias Sociales y Jurídicas se debería concentrar la atención o la intervención sobre las reubicaciones en el primer año de estudios, después de la primera matrícula, mediante acciones más focalizadas en acompañar o actuar en la incorporación a la universidad; en cambio, en los casos de las ramas de Arquitectura e Ingeniería y de Ciencias de la Salud el punto de atención se traslada más hacia años posteriores a la incorporación universitaria.

La agrupación de los datos y las actuaciones diferenciadas por ramas de conocimiento nos parecen imprescindibles puesto que aportan escenarios muy diferentes. Según los datos para el total de universidades públicas españolas (Anuario de Indicadores Universitarios del Sistema Integrado de Información Universitaria, 2016), la tasa de cambio de estudio en la rama de Ciencias supera el $16 \%$ y en casi un $13 \%$ en Ingeniería y Arquitectura y supera el $11 \%$ en Artes y Humanidades $y$ en Ciencias Sociales y Jurídicas, en Ciencias de la Salud no llega al $8 \%$.

Como comentario final, podemos afirmar que este trabajo constata que no podemos considerar la institución universitaria de manera isomorfa, puesto que las titulaciones que la integran presentan comportamientos divergentes en cuanto a la configuración de las trayectorias académicas, un hecho que también se muestra en la agrupación según ramas de conocimiento. Este trabajo apunta la necesidad de estudiar estos resultados en diferentes instituciones para comprobar si se pueden generalizar.

\section{Referencias}

Ariño, A., \& Llopis, R. (2011). ¿Universidad sin clases? Condiciones de vida de los estudiantes universitarios en España 
(Eurostudent IV). Madrid: Ministerio de Educación.

Bethencourt, J.; Cabrera, L.; Hernández, J.; Álvarez, P. \& González, M. (2008). Variables psicológicas y educativas en el abandono universitario. EJREP, 6, 339-356. http://www.investigacion-

psicopedagogica.org/revista/new/LeerArticul o.php

Cabrera, L., Bethencourt, J. T., Álvarez Pérez, P. y González Afonso, M. (2006). El problema del abandono de los estudios universitarios. RELIEVE, 12(2), art. 1. doi: http://doi.org/10.7203/relieve.12.2.4226

Fachelli, S. \& Navarro-Cendejas, J. (2015). Relación entre origen social e inserción laboral de los graduados universitarios. RELIEVE, 21(2), art. 2. doi: https://doi.org/10.7203/relieve.21.2.7812

García-Ros, R. \& Pérez-González, F. (2011). Validez predictiva e incremental de las habilidades de autorregulación sobre el éxito académico en la universidad. Revista de Psicodidáctica, 16, 231-250. doi: http://dx.doi.org/10.1387/RevPsicodidact.1002

Glogowska, M., Young, P., \& Lockyer, L. (2007). Should I go or should I stay? A study of factors influencing students' decisions on early leaving. Active Learning in Higher Education, 8, 63-77, doi: https://doi.org/10.1177/1469787407074115

Lassibille, G., \& Navarro, L. (2009). Tracking students' progress through the Spanish university school sector. Higher Education, 58(6), 821-839.

Ministerio de Educación, Cultura y Deporte (2016). Anuario de indicadores universitarios. Recuperado de https://www.mecd.gob.es/dms/mecd/servicio s-al-ciudadano-

mecd/estadisticas/educacion/universitaria/dat os-cifras/datos-y-cifras-SUE-2015-16-web.pdf

Rodrigo, M., Molina, G., García-Ros, R. \& Pérez-González, F. (2012). Efectos de interacción en la predicción del abandono en los estudios de Psicología. Anales de Psicología, 28, 113-119. http://revistas.um.es/analesps/article/view/14 $\underline{0592 / 126682}$

Troiano, H. \& Elias, M. (2013). University access and after: explaining the social composition of degree programmes and the contrasting expectations of students. Higher Education, 67(5), 637-654. doi: https://doi.org/10.1007/s10734-013-9670-4

Villar Aguilés, A. (2010). Del abandono de estudios a la reubicación universitaria. Revista de la Asociación de Sociología de la Educación, vol. 3, 2: 267-283, doi: http://doi.org/10.7203/RASE.3.2.8705

Villar, A., Vieira, M.M., Hernàndez, F., Almeida, A. (2012). Más que abandono de estudios, trayectorias de reubicación universitaria. Aproximación comparada al caso español y portugués. Revista Lusófona de Educação, 21, 139-162. http://revistas.ulusofona.pt/index.php/rleduca cao/article/view/3085

\section{Nota}

Este artículo ha sido escrito en el marco del proyecto "Trayectorias, abandonos y cambios de estudios en la Universidad de Valencia” y cuenta con el apoyo institucional y financiero del Vicerectorat d'Estudis de Grau de la Universitat de València. Una versión previa de este artículo fue presentada en el Congreso Español de Sociología (julio de 2016). 
Villar Aguilés, Alicia; Hernàndez i Dobon, Francesc Jesús \& García Ros, Rafael (2017). Reubicándose en la universidad. Propuesta de una tasa de reubicaciones a partir de un estudio de trayectorias educativas. RELIEVE, 23(1), art. 5. doi: http://doi.org/10.7203/relieve.23.1.9059

\begin{tabular}{|c|c|}
\hline Autores / Authors & To know more / Saber más \\
\hline Villar-Aguilés, Alícia (alicia.villar@uv.es). & ORCID \\
\hline $\begin{array}{l}\text { Profesora del Departamento de Sociología y Antropología Social de la } \\
\text { Universidad de Valencia. Sus líneas de investigación están relacionadas con los } \\
\text { análisis sobre las transformaciones de los perfiles de estudiantes universitarios, } \\
\text { las trayectorias y transiciones educativas y profesionales. Dirección postal: } \\
\text { Departament de Sociologia i Antropologia Social. Universitat de València. } \\
\text { Avinguda dels Tarongers 4b, } 46022 \text { Valencia (España) }\end{array}$ & $\underline{0000-0003-1748-0234}$ \\
\hline Hernàndez i Dobon, Francesc Jesús (francesc.j.hernandez@uv.es). & ORCID \\
\hline $\begin{array}{l}\text { Profesor y actual director del Instituto de Creatividad e Innovaciones Educativas } \\
\text { de la Universidad de Valencia. Sus líneas de investigación están relacionadas con } \\
\text { el estudio de las desigualdades educativas y sociales, los indicadores educativos y } \\
\text { las trayectorias educativas. Dirección postal: Departament de Sociologia i } \\
\text { Antropologia Social. Universitat de València. Avinguda dels Tarongers 4b, } 46022 \\
\text { Valencia (España) }\end{array}$ & $\underline{0000-0001-5229-2998}$ \\
\hline García-Ros, Rafael (rafael.garcia@uv.es). & ORCID \\
\hline $\begin{array}{l}\text { Profesor y actual director del Departamento de Psicología Evolutiva y de la } \\
\text { Educación de la Universidad de Valencia. Sus líneas de investigación se centran } \\
\text { en el aprendizaje autorregulado, en la evaluación orientada al aprendizaje, y en } \\
\text { el diseño de recursos y propuestas de intervención dirigidas mejorar el acceso a } \\
\text { la universidad. Dirección postal: Departament de Psicologia Evolutiva i de } \\
\text { l’Educació. Universitat de València. Facultat de Psicologia. Av. Blasco Ibáñez, } \\
\text { 21, } 46010\end{array}$ & $\underline{0000-0001-9486-1001}$ \\
\hline
\end{tabular}

\section{RELIEVE}

\section{Revista ELectrónica de Investigación y EValuación Educativa E-Journal of Educational Research, Assessment and Evaluation}

[ISSN: 1134-4032]

(C) Copyright, RELIEVE. Reproduction and distribution of this articles it is authorized if the content is no modified and their origin is indicated (RELIEVE Journal, volume, number and electronic address of the document).

(C) Copyright, RELIEVE. Se autoriza la reproducción y distribución de este artículo siempre que no se modifique el contenido y se indique su origen (RELIEVE, volumen, número y dirección electrónica del documento). 